1

2

\title{
Infrared absorptions as indicators for Pseudomonas aeruginosa and Acinetobacter baumannii
}

Masato Yamamoto*1, Satoru Arata ${ }^{2}$, Kunihiko Fukuchi ${ }^{3}$, Hidehiko Honda ${ }^{1}$, Hirokazu Kobayashi ${ }^{1}$,

Masahiro Inagaki ${ }^{1}$

${ }^{1}$ Department of Chemistry, College of Arts and Sciences, Showa University, 4562 Kamiyoshida,

Fujiyoshida-city, Yamanashi 403-0005, Japan

${ }^{2}$ Center for Biotechnology, Showa University, 1-5-8 Hatanodai, Shinagawa-ku, Tokyo 142-8555,

Japan

${ }^{3}$ Graduate School of Health Sciences, Showa University, 1-5-8 Hatanodai, Shinagawa-ku, Tokyo 142-

8555, Japan

*Corresponding author

Email: yama@cas.showa-u.ac.jp (MY) 


\section{Abstract}

20 There is clinical demand for simple and contact-free diagnosis techniques in medical practice. This

21 study shows that the infrared absorptions of volatile metabolites can be used to distinguish between

the air around Pseudomonas aeruginosa, Acinetobacter baumannii, other bacteria, and normal room air. Gas samples were collected from the air surrounding single and mixed laboratory cultures, and

preliminary data on human breath samples are also presented. The infrared spectra of a variety of

position of the key bands. Hence, it is not necessary to specify the molecular species of biomarkers.

This work also shows that discrimination rates can be improved by observing additional infrared

absorptions caused by different characteristic volatile molecules. The significance of this work is that

the specific wavenumber positions of the key bands that allow the application of infrared lasers are

provided. As a result, it is considered that Pseudomonas aeruginosa and Acinetobacter baumannii can

approach could be used in future applications to identify infections in healthcare settings.

\section{Introduction}

Biomarkers are characteristic molecules produced by biological processes. The biomarkers found 
37

representative small molecules such as $\mathrm{CO}, \mathrm{CO}_{2}, \mathrm{NO}$, and $\mathrm{NO}_{2}$ have been the target of high-sensitivity

infrared laser spectroscopy measurements [6-9]. Clinical applications of these techniques have been

proposed [10-12].

To apply an infrared laser, it is necessary to adjust the oscillation wavenumber (the reciprocal of the wavelength) to a specific value. A molecule produces many peaks, but the wavenumber indicates the position of one strong peak on the horizontal axis of the infrared spectrum. Infrared lasers can be used to detect changes in the concentration (partial pressure) of some target volatile biomarkers. In particular, it has been shown that changes in the concentration of ethane [13] and ethanol [14] can be detected with ultra-high sensitivity, in addition to the molecules $\mathrm{CO}, \mathrm{CO}_{2}, \mathrm{NO}$, and $\mathrm{NO}_{2}$.

High resolution infrared spectra can be obtained for a wide variety of gas samples. By analyzing the infrared absorption peaks it is possible to identify the key band, the infrared absorption peak that serves as the target index of a biomarker. There are two criteria for defining a key band. First, it must appear in a "window" region where there are no unrelated strong peaks; this means the intensity of the key band can be observed clearly. Second, the peak position of the wavenumber must be accurately determined in a range close to the linewidth of the infrared laser.

The intensity and wavenumber position of the key band correspond to quantitative information about the concentration (partial pressure) of key volatile metabolites (VMs) or volatile organic compounds (VOCs). This information is the index equivalent to surveillance markers and can be used 
to distinguish between bacterial species using the surrounding air.

56 In recent years, several reports [15-19] have classified bacteria based on infrared spectra measured

57 using Fourier-transform infrared (FTIR) spectroscopy. In these studies, samples were prepared by

the bacteria. The infrared spectra were measured with a general resolution of $4 \mathrm{~cm}^{-1}$ by directly

irradiating bacteria with infrared light and transmitting or reflecting the light.

In this study, infrared spectra have been recorded for a wide variety of gas samples including gases peaks) can be found. Therefore, the oscillation frequency (wavenumber) of the infrared laser can be fine-tuned in a limited area, which makes it possible to detect bacteria with high sensitivity without identifying the molecular species as the target index markers. 
absorption peaks in the region of $2000-2300 \mathrm{~cm}^{-1}$. However, specifying the molecular structure of the

key VOC does not automatically mean it can be found because the key band must appear in the

"window" region so that the intensity can be read. The condition is confirmed only by comparison

with many infrared spectra of other gas samples.

The next condition is whether the accuracy of the key-band position is in a range that facilitates the

application of an infrared laser. Experimental results for a pure substance and the theoretical

calculations for a molecule, such as normal vibration analysis for the most stable structure, may be

used to predict candidates for the key bands. However, gas samples contain a mixture of substances

including $\mathrm{H}_{2} \mathrm{O}, \mathrm{CO}_{2}$, and other atmospheric components, which could affect the key-band position by

binding to the key molecule. Furthermore, the infrared spectra may potentially reflect molecular

aggregates, clusters, and small particles suspended in the atmosphere, which have a different structure

than that suggested by the GCMS. A key molecule does not always give the key bands at the same position, intensity, and width as the prediction.

This study aims to identify the key bands that characterize the air around Pseudomonas aeruginosa

and Acinetobacter baumannii. These are typical drug-resistant bacteria that are associated with

nosocomial infections, so there is urgent need for simple and contact-free detection techniques that 
91 Specifically, the wavenumber position of the key bands for VOCs or VMs emitted by the bacteria are

92 recorded at a high resolution of $0.5 \mathrm{~cm}^{-1}$, which makes it possible to determine the type of bacteria

93 without contact. Furthermore, this work aims to demonstrate that the high intensity infrared absorption

94 peak that appears at a specific wavenumber position can be used as an indicator for nearby bacterial

95 growth, even if the molecular structure of the characteristic VOC(s) is not specified.

96 The positions of the key bands are found from the experimental results (the accumulation of infrared

97 spectra in the region of $550-7000 \mathrm{~cm}^{-1}$ ) for various mixed substances, as a result of the search and

selection. Therefore, the key substance is not limited to a single molecule in the most stable structure.

This differs from the previous studies because any suspended substances shown by the infrared

102 Methods

\section{Preparation of gas samples}

104 Gas samples were obtained from the air surrounding cultured bacteria. The gases were aspirated into gas bags (smart bag PA, smart bag 2F, Tedlar bag or ANALYTIC-BARRIER bag, GL Sciences) 
109 cultured in sheep's blood agar at $37^{\circ} \mathrm{C}$ for 30 to $40 \mathrm{~h}$. The details are summarized in Table 1. M1, M2,

110 M3, and M4 indicate mixed gas samples obtained from plural types of bacteria cultured in a nutrient

111 agar at $37^{\circ} \mathrm{C}$. For comparison, normal room air, human breath, and air from the vicinity of the culture

112 medium alone were also obtained and measured. The human breath was blown into a polyethylene

113 zipper bag.

114

115

116

117

118

119

120

121

122

123

124

125 
Table 1. Details of the gas samples.

\begin{tabular}{|c|c|c|c|c|c|c|}
\hline $\begin{array}{l}\text { Air } \\
\text { around } \\
\text { bacteria }\end{array}$ & Genus species & ID & Strain/Origin & $\begin{array}{l}\text { Number } \\
\text { of } \\
\text { samples }\end{array}$ & $\begin{array}{l}\text { Total number } \\
\text { of } \\
\text { measurements }\end{array}$ & $\begin{array}{l}\text { Incubation } \\
\text { time (h) }\end{array}$ \\
\hline \multirow{7}{*}{$\begin{array}{l}\text { Standard } \\
\text { strains }\end{array}$} & Escherichia coli & $E c$ & $\begin{array}{l}\text { type strain (NBRC } \\
3972)\end{array}$ & 2 & $8(1+7)$ & 32 \\
\hline & Staphylococcus aureus & $S a$ & $\begin{array}{l}\text { type strain (NBRC } \\
13276)\end{array}$ & 2 & $8(4+4)$ & 39 \\
\hline & Pseudomonas aeruginosa & Pal & $\begin{array}{l}\text { type strain } \\
\text { (NBRC113275) }\end{array}$ & 2 & $11(5+6)$ & 38.5 \\
\hline & \multirow{4}{*}{$\begin{array}{l}\text { plural cluture plates in a } \\
\text { chamber for the mixed gas } \\
\text { collection }\end{array}$} & $M 1$ & $\mathrm{Ec}+\mathrm{Sa}$ & 2 & $19(7+12)$ & $38^{*}$ \\
\hline & & $M 2$ & $\mathrm{Ec}+\mathrm{Pa} 1$ & 2 & $17(3+14)$ & $38 *$ \\
\hline & & M3 & $\mathrm{Sa}+\mathrm{Pa} 1$ & 2 & $11(3+8)$ & $38 *$ \\
\hline & & M4 & $\mathrm{Ec}+\mathrm{Sa}+\mathrm{Pa} 1$ & 2 & $12(5+7)$ & $38 *$ \\
\hline \multirow{12}{*}{$\begin{array}{l}\text { Hospital } \\
\text { isolates }\end{array}$} & \multirow{2}{*}{ Pseudomonas aeruginosa } & $\mathrm{Pa} 2$ & hospital enviroment 1 & 2 & $10(5+5)$ & 39 \\
\hline & & $\mathrm{Pa} 3$ & hospital enviroment 2 & 2 & $10(5+5)$ & 39 \\
\hline & \multirow{5}{*}{ Acinetobacter baumannii } & $A b 1$ & clinical isolate 1 & 2 & $11(5+6)$ & 38.5 \\
\hline & & $A b 2$ & clinical isolate 2 & 2 & $10(5+5)$ & 38 \\
\hline & & $A b 3$ & clinical isolate 3 & 2 & $10(5+5)$ & 38 \\
\hline & & $A b 4$ & clinical isolate 4 & 2 & $11(5+6)$ & 39 \\
\hline & & $A b 5$ & clinical isolate 5 & 2 & $11(5+6)$ & 39 \\
\hline & Enterobacter aerogenes & Ena & clinical isolate & 2 & $10(4+6)$ & 37.5 \\
\hline & Enterobacter cloacae & Enc & clinical isolate & 2 & $8(1+7)$ & 39 \\
\hline & Klebsiella pneumoniae & $K p$ & clinical isolate & 2 & $8(1+7)$ & 32 \\
\hline & \multirow{2}{*}{ Streptococcus pneumoniae } & $S p 1$ & clinical isolate 1 & 2 & $10(4+6)$ & 37.5 \\
\hline & & $S p 2$ & clinical isolate 2 & 2 & $8(4+4)$ & 37.5 \\
\hline & & & & & & $*$ means a \\
\hline \multirow{4}{*}{$\begin{array}{l}\text { Reference } \\
\text { samples }\end{array}$} & \multirow{2}{*}{$\begin{array}{l}\text { air around the culture } \\
\text { medium without bacterial } \\
\text { breeding }\end{array}$} & \multicolumn{2}{|c|}{ blood agar } & 2 & $8(4+4)$ & nutrient agar \\
\hline & & \multicolumn{2}{|c|}{ nutrient agar } & 3 & $8(2+3+3)$ & $\begin{array}{l}\text { for the culture } \\
\text { medium, the }\end{array}$ \\
\hline & room air & \multicolumn{3}{|c|}{ taken from 5 places } & 146 & others are \\
\hline & human breath & \multicolumn{3}{|c|}{ taken from 3 health people } & 136 & $\begin{array}{c}\text { sheep blood } \\
\text { agar. }\end{array}$ \\
\hline
\end{tabular}




\section{Measurements}

132 The gas in each bag was introduced into a gas cell (light path length $10 \mathrm{~m}$ ) installed in an FTIR

spectrometer (Bruker VERTEX 70). Overall, 365 infrared (IR) spectra for the various gas samples in

134 Table 1 were measured in the range of $550-7500 \mathrm{~cm}^{-1}$ at atmospheric pressure with a resolution of 0.5

$\mathrm{cm}^{-1}$.

\section{Data analysis}

137 Microsoft EXCEL was used for data processing and analysis. The details of the infrared absorption

138 peaks that appeared in the IR spectra were obtained and the peak intensities were measured and

139 compared. The peak intensity was calculated by subtracting the absorbance at the base point from that

140 at the absorption peak. Peak and base pairs were found around the peak positions where the target

141 group showed strong absorption. The mean and variance of the absorption intensity for the reference

142 group were compared with the target group. Peak and base pairs with high discrimination rates were

143 extracted for each, pair and high discrimination rates were achieved for P. aeruginosa and A.

144 baumannii.

\section{Ethics statement}

146 This study was approved by the research ethics committee of Showa University School of Medicine 


\section{Results and discussion}

\section{Pseudomonas aeruginosa $(\mathrm{Pa})$}

150 Fig 1 shows the IR spectra at approximately $2213 \mathrm{~cm}^{-1}$ for the air surrounding the eight types of

151 cultured bacteria and the normal room air. These nine spectra are offset and aligned. The vertical axis

152 indicates absorbance and the absorption peak appears in the upward direction. In this region of the IR

153 spectra, a strong peak can be observed at $2213.2 \mathrm{~cm}^{-1}$, which is characteristic of $P a$.

room air. The 9 spectra are offset and aligned in the following order from the top: Pseudomonas

Enterobacter cloacae (Enc), Enterobacter aerogenes (Ena), Acinetobacter baumannii (Ab),

Streptococcus pneumoniae $(\mathrm{Pa})$, and room air. 
165 the base point from that at the peak. This value was used as the intensity of the absorption peak at

$1662213.2 \mathrm{~cm}^{-1}$ assigned to the VM from $\mathrm{Pa}$. In all of the spectra, the two fixed positions for the assumed

167 peak and base do not actually correspond to the wavenumbers at the local maximum and minimum,

168 respectively.

169 The absorption peaks at $2213.2 \mathrm{~cm}^{-1}$ and $778.4 \mathrm{~cm}^{-1}$ were taken as the index of $P a$. The absorption

170 intensity of the peak at $778.4 \mathrm{~cm}^{-1}(\mathrm{P}$ at 778.4$)$ is the difference from the value for the base point at

$171 \quad 778.6 \mathrm{~cm}^{-1}(\mathrm{~B}$ at 778.6$)$.

172 Fig 2 shows the results for discrimination of $\mathrm{Pa}$ using two intensities as the $\mathrm{Pa}$ indexes. The

173 subtracted value, $\mathrm{P}$ at 778.4 - B at 778.6, is shown on the horizontal $(x)$ axis, and that of P at 2213.2 -

$174 \quad \mathrm{~B}$ at 2213.7 is shown on the vertical $(y)$ axis. The gas samples that included VMs from $P a$ (filled grey

175 squares $\square)$ are grouped in the upper right region, which is delimited by two dotted lines $(x>0$ and $y$

$176>0.003)$. All of the other cases are distinguished. The 365 points in Fig 2 correspond to the values

177 obtained for the 365 spectra from the various gas samples shown in Table 1 . Open circles $\bigcirc$ indicate

178 the values obtained from human breath samples. The infrared spectra of human breath were measured

179 under the same conditions as the air samples for cultured bacteria and they have been stored for analysis. The results will be published in later works. 
184 Grey squares $\square$, the values obtained from the gas samples that included VMs from $\mathrm{Pa}$; open squares

$185 \square$, the other 7 types of bacteria; black triangles $\boldsymbol{\Lambda}$, air from the vicinity of the culture medium alone;

186 open triangles $\triangle$, the normal room air; and open circles $\bigcirc$, human breath. $M 2, M 3$, and $M 4$ indicate

187 the data from the mixed gas samples that included VMs from $\mathrm{Pa}$ and the other type(s) of bacteria, as

188 shown in Table 1.

190 The values on the $x$ and $y$ axes reflect the infrared absorption peaks of the VMs specific to $P a$.

191 According to Lambert-Beer's law, the infrared absorption intensity indicated by absorbance is

proportional to the concentration or partial pressure. The gas samples contain multiple unknown

Fig 2 do not show a strong linear relationship. However, Fig 2 shows that $P a$ can be clearly detected

197 by infrared light with specific wavenumbers passing through the surrounding air.

198 GCMS has been used to show that methyl thiocyanate and benzonitrile are characteristic VMs of 
201 absorption peaks in the same region. However, this study does not exclude other VMs that may

202 produce the index peak, because FTIR does not require the ionization of VMs and can provide

203 information about unstable VMs, unlike GCMS.

\section{Acinetobacter baumannii (Ab)}

Fig 3 shows the result for discrimination of $A b$ using two intensities as the $A b$ indexes. One is the

211 The $A b$ index, the intensity of the absorption peak at $2982.3 \mathrm{~cm}^{-1}$ is in the $\mathrm{CH}$ stretching vibration

212 region; this indicates that the causative VM molecule contains a hydrocarbon structure characteristic

213 of $A b$. The other $A b$ index observed at $1215.0 \mathrm{~cm}^{-1}$ is derived from another VM and the ratio of these

214 VMs is specific to $A b$, as shown in Fig 3.

216 Fig 3. Discrimination of Acinetobacter baumannii $(A b)$ with two $A b$ indexes, $P$ at 1215.0 - B at

$217 \quad 1214.5$ and $P$ at 2982.3 - B at 2982.9, shown on the horizontal $(x)$ and vertical $(y)$ axes, 
219 Acinetobacter baumannii $(A b)$; black triangles $\boldsymbol{\Delta}$, air from the vicinity of the culture medium

220 alone; black diamonds $\bullet$, Escherichia coli $(E c)$; open squares $\square$, the other 6 types of bacteria

221 in Table 1 except $A b$ and $E c$, open triangles $\triangle$, the normal room air; and open circles $\bigcirc$, human 222 breath

224 In Fig 3, the $A b$ area $(y>3.76 x$ and $y<9.65 x$, a triangular zone between the two dotted lines)

225 overlaps with other plots in the lower left corner. This is due to the detection sensitivity and the

226 wavenumber resolution of the FTIR spectroscopy and it could be improved by adopting an infrared

227 laser system. The $A b$ area also overlaps with the areas for Escherichia coli $(E c, \bullet$ filled black diamond)

228 and the blood agar medium ( $\boldsymbol{\Delta}$ filled black triangle in the upper left), which causes the $A b$

229 discrimination rate to deteriorate.

230 Two additional indices of $A b$ were found, as shown in Fig 4, indicating there was an infrared

231 absorption peak at $4768.7 \mathrm{~cm}^{-1}(\mathrm{P}$ at $4768.7-\mathrm{B}$ at 4768.1$)$ on the $x$ axis and $5353.8 \mathrm{~cm}^{-1}(\mathrm{P}$ at 5353.8

$232-\mathrm{B}$ at 5354.4) on the $y$ axis. The two dotted lines $(y=-188 x$ and $y=78 x)$ separate the points for $A b$,

$233 E c$, and culture medium only. By combining the information from Figs 3 and 4, the rate of $A b$

234 discrimination can be increased. 


\section{Application and data processing}

251 Infrared lasers can be applied to detect the key VMs of $P a$ and $A b$ using three steps: 
(3) a discrimination program with consideration of the optical path length.

256 It is worth noting that the gas samples in this experiment were under atmospheric pressure. This

257 work assumes that the atmospheric pressure during any application is the same as at the experiment site.

$$
A=-\log (T)=-\log \left(I_{\text {sample }} / I_{\text {blanc }}\right)
$$

addition,

$$
=-\log \left(\left(I_{\text {sample at } \mathrm{P}} / I_{\text {blanc at } \mathrm{P}}\right) /\left(I_{\text {sample at B }} / I_{\text {blanc at B }}\right)\right)
$$

where $A_{\text {at }}$ and $A$ at B are the absorption values at $\mathrm{P}$ and $\mathrm{B}$, respectively. 


$$
A_{\text {at } \mathrm{P}}-A_{\text {at B }} \approx-\log \left(I_{\text {at }} / I_{\text {at }}\right)
$$

274 where $I_{\text {at P }}$ and $I_{\text {at B }}$ are the raw intensity values at $\mathrm{P}$ and B, respectively, which can be observed by

275 the infrared laser system.

276 The value of $A$ at $\mathrm{P}-A$ at B reflects to the partial pressure (concentration) of a key VM. Two sets of

$277 A_{\text {at P }}$ and $A_{\text {at B }}$ for two key VMs can provide information about their component ratios. An appropriate

278 combination of these sets can be used to show the characteristics of the gas sample more clearly.

279 The discriminant in Figs $2-4$ is based on the measurements obtained using a gas cell with an optical

280 path length of $10 \mathrm{~m}$. According to Lambert-Beer's law, the absorbance is proportional to the optical

281 path length under the same pressure. If the optical path length is $\mathrm{L} \mathrm{m}$, which is part of the discriminant,

then $y>0.003$ shown in Fig 2 becomes $y>0.003 \times \mathrm{L} / 10$. In addition, $x>0$ in Fig $2, y=9.65 x$ and $y$

$=3.76 x$ in Fig 3, and $y=-188 x$ and $y=78 x$ in Fig 4 are linear functions through the origin that are not

dependent on the optical length.

Since the absorption intensity is proportional to the optical path length, the longer the optical path

length, the better the sensitivity. For this reason, infrared laser systems have an advantage over other

methods for analyzing low-density gas samples. Most biogas samples are obtained non-invasively.

Further, infrared light generally has low energy and is non-destructive. Therefore, it would be realistic 
291 the size of the space to be examined, multiple reflections can be used to increase the optical path length

292 even in a small space, and to achieve high sensitivity.

\section{Conclusion}

This study demonstrated that $P$. aeruginosa and $A$. baumannii can be detected using infrared lights

with specific wavenumbers that pass through the surrounding air. The discrimination rates can be

be used in the application of infrared lasers for continuous monitoring of $P$. aeruginosa and $A$.

baumannii with high sensitivity.

300 The next step involves fine-tuning of the oscillating wavenumber of the infrared laser around the

301 values reported in this study. The fine adjustment will be performed by finding a wavenumber position

302 with the best discrimination rate in consideration of the line width of the infrared laser. As the result, 
bioRxiv preprint doi: https://doi.org/10.1101/2020.03.25.007591; this version posted March 25, 2020. The copyright holder for this preprint

(which was not certified by peer review) is the author/funder, who has granted bioRxiv a license to display the preprint in perpetuity. It is made available under aCC-BY 4.0 International license.

\section{Acknowledgments}

311 We would like to thank Editage for English language editing. This work was supported by JSPS

312 KAKENHI (Grant Number JP26462957) and Research Fund of Showa University, College of Arts

313 and Sciences (2015).

314

315 


\section{References}

317 [1] Amann A, Smith D. Volatile Biomarkers: Non-Invasive Diagnosis in Physiology and Medicine.

318 Science Direct, Elsevier; 2013.

319 [2] Jia Z, Patra A, Kutty VK, Venkatesan T. Critical Review of Volatile Organic Compound Analysis

320 in Breath and In Vitro Cell Culture for Detection of Lung Cancer. Metabolites. 2019; 9:52.

321 [3] Saalberg Y, Wolff M. VOC breath biomarkers in lung cancer. Clinica Chimica Acta. 2016; 459:5.

322 [4] Wilson AD. Advances in Electronic-Nose Technologies for the Detection of

323 Volatile Biomarker Metabolites in the Human Breath. Metabolites. 2015; 5:140.

324 DOI: $10.3390 /$ metabo5010140.

325 [5] Sethi S, Nanda R, Chakraborty T. Clinical Application of Volatile Organic Compound Analysis

326 for Detecting Infectious Diseases. Clin Microbiol Rev. 2013; 26(3):462.

327 [6] Popp A, Müller F, Kühnemann F, Schiller S, von Basum G, Dahnke H, et al. Ultra-sensitive mid-

328 infrared cavity leak-out spectroscopy using a cw optical parametric oscillator. Appl Phys B. 2002;

$329 \quad 75(6-7): 751$.

330 [7] Engel GS, Drisdell WS, Keutsch FN, Moyer EJ, Anderson JG. Ultrasensitive near-infrared

331 integrated cavity output spectroscopy technique for detection of $\mathrm{CO}$ at $1.57 \mu \mathrm{m}$ : new sensitivity limits

332 for absorption measurements in passive optical cavities. Appl Opt. 2006; 45(36):9221.

333 [8] Shorter JH, Nelson DD, McManus JB, Zahniser MS, Sama S, Milton DK. Clinical study of 
334 multiple breath biomarkers of asthma and $\mathrm{COPD}\left(\mathrm{NO}, \mathrm{CO}_{2}, \mathrm{CO}\right.$ and $\left.\mathrm{N}_{2} \mathrm{O}\right)$ by infrared laser

335 spectroscopy. J Breath Res. 2011; 5(3):037108.

336 [9] Mandon J, Högman M, Merkus PJFM, van Amsterdam J, Harren FJM, Cristescu SM. Exhaled

337 nitric oxide monitoring by quantum cascade laser: comparison with chemiluminescent and

338 electrochemical sensors. J Biomed Opt. 2012; 17(1):017003.

339 [10] Mürtz M, Halmer D, Horstjann M, Thelen S, Hering P. Ultra sensitive trace gas detection for

340 biomedical applications. Spectrochim Acta A Mol Biomol Spectrosc. 2006; 63(5)L:963. DOI:

$341 \quad 10.1016 / j . s a a .2005 .11 .015$.

342 [11] Kim S-S, Young C, Vidakovic B, Gabram-Mendola SGA, Bayer CW, Mizaikoff B. Potential and

343 Challenges for Mid-Infrared Sensors in Breath Diagnostics. IEEE Sens. 2010; 10(1):145. DOI:

$344 \quad 10.1109 / J$ SEN.2009.2033940.

345 [12] Henderson B, Khodabakhsh A, Metsälä M, Ventrillard I, Schmidt FM, Romanini D, et al. Laser

346 spectroscopy for breath analysis: towards clinical implementation. Appl Phys B. 2018; 124:161. DOI:

$347 \quad 10.1007 / \mathrm{s} 00340-018-7030-\mathrm{x}$.

348 [13] von Basum G, Dahnke H, Halmer D, Hering P, Mürtz M. Online recording of ethane traces in

349 human breath via infrared laser spectroscopy. J Appl Physiol. 2003; 95(6):2583. DOI:

10.1152/japplphysiol.00542.2003.

351 [14] Jones AW, Andersson L. Determination of ethanol in breath for legal purposes using a five-filter 
352 infrared analyzer: studies on response to volatile interfering substances. J Breath Res. 2008;

354 [15] Fischer G, Braun S, Thissen R, Dott W. FT-IR spectroscopy as a tool for rapid identification and

355 intra-species characterization of airborne filamentous fungi. J Microbiol Methods. 2006; 64:63.

356 [16] Taha M, Hassan M, Essa S, Tartor Y. Use of Fourier transform infrared spectroscopy (FTIR)

357 spectroscopy for rapid and accurate identification of Yeasts isolated from human and animals. Int $\mathbf{J}$

$358 \quad$ Vet Sci Med. 2013; 1:15.

359 [17] Maity JP, Kar S, Lin C-M, Chen C-Y, Chang Y-F, Jean J-S, et al. Identification and discrimination

360 of bacteria using Fourier transform infrared spectroscopy. Spectrochim Acta A Mol Biomol Spectrosc. as a tool to characterize molecular composition and stress response in foodborne pathogenic bacteria. 


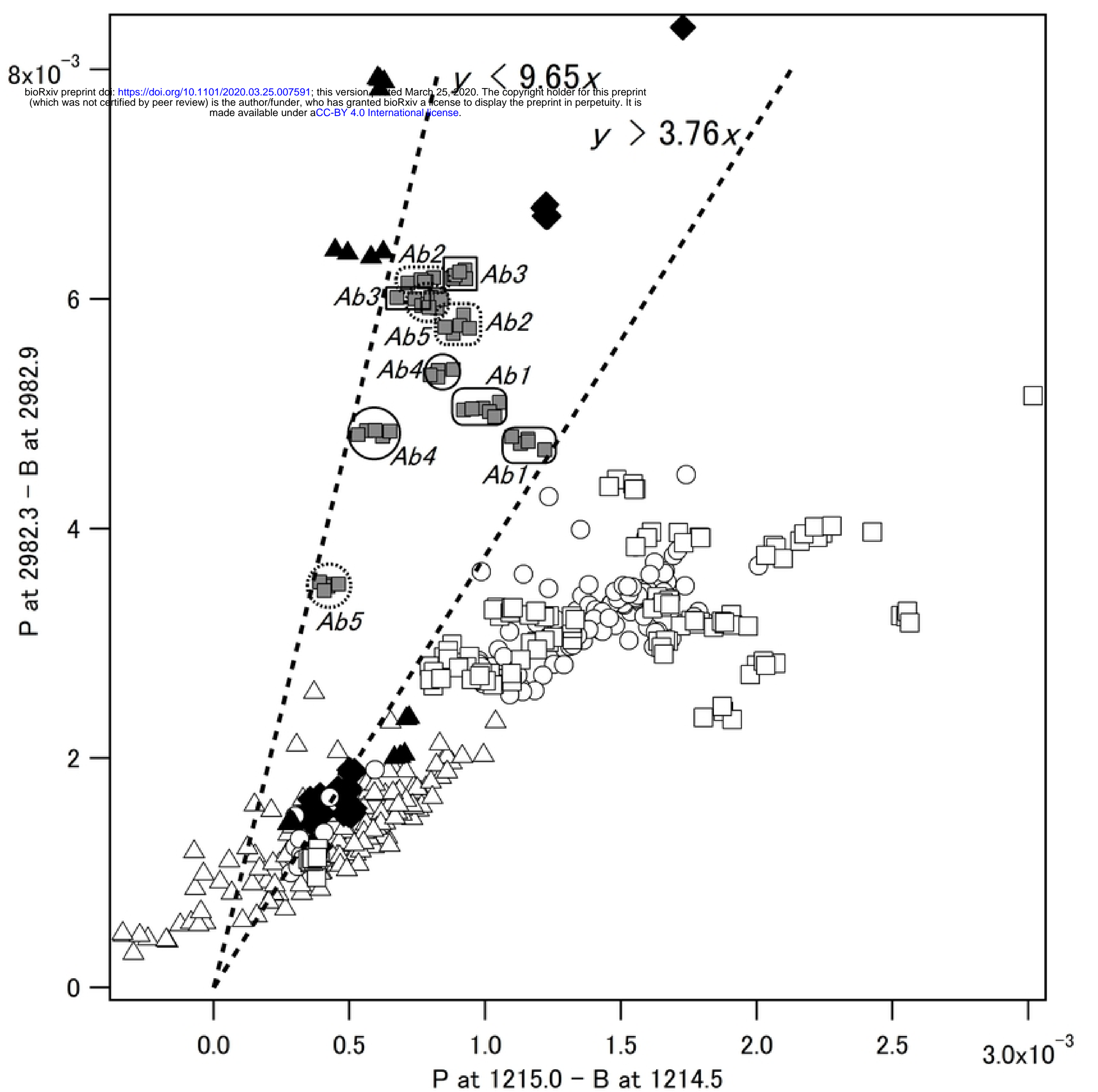

Figure 3 


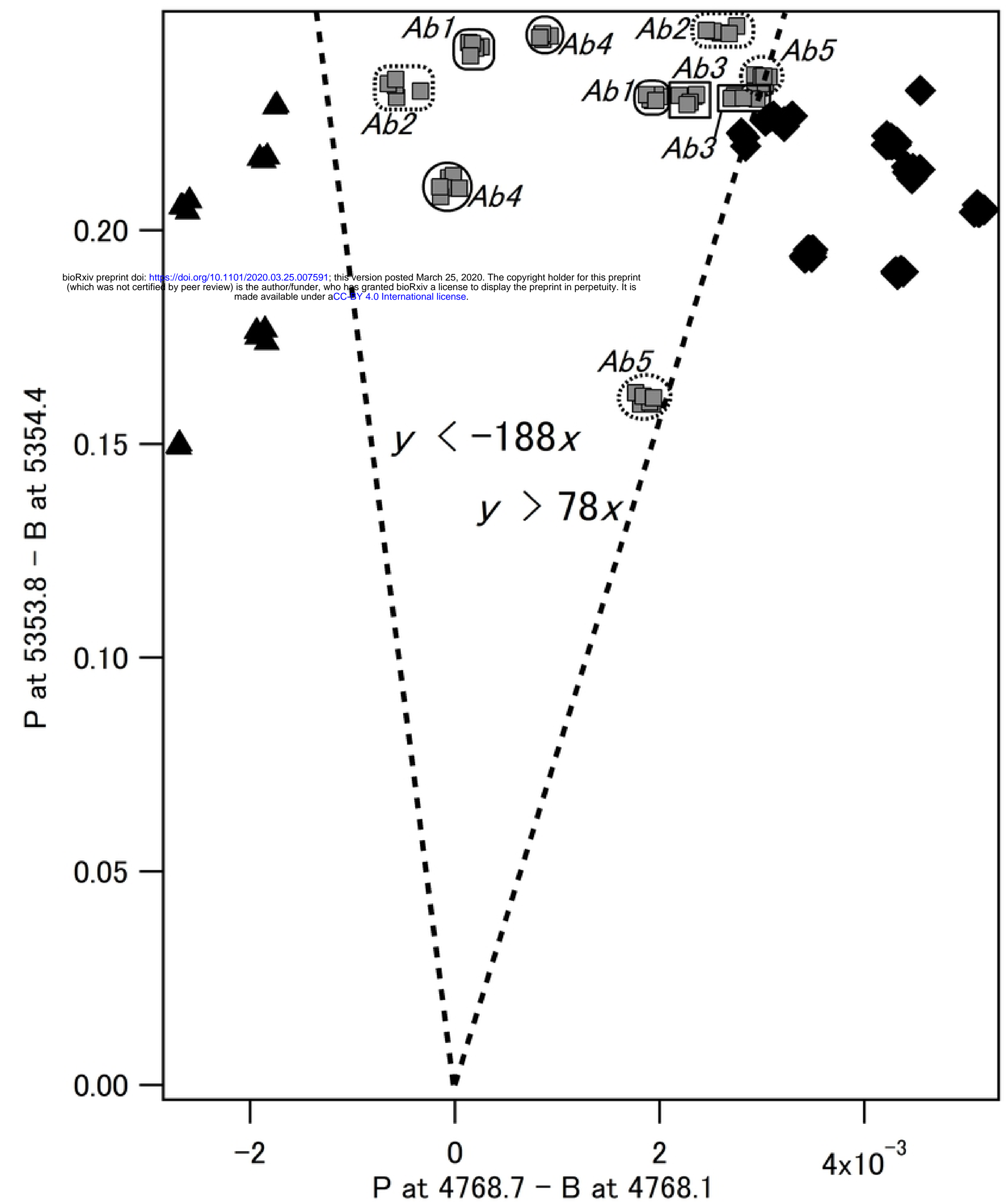

Figure 4 